\title{
Cosmic axion force
}

\author{
Dongok Kim๑, ${ }^{1,2}$ Younggeun Kim๑, ${ }^{1,2}$ Yannis K. Semertzidis $\odot,{ }^{2,1}$ Yun Chang Shin $\odot,{ }^{2}$ and Wen Yin $\odot^{3,4}$ \\ ${ }^{1}$ Department of Physics, Korea Advanced Institute of Science and Technology, \\ Daejeon 34141, Republic of Korea \\ ${ }^{2}$ Center for Axion and Precision Physics Research, Institute for Basic Science, \\ Daejeon 34051, Republic of Korea \\ ${ }^{3}$ Department of Physics, Tohoku University, Sendai, Miyagi 980-8578, Japan \\ ${ }^{4}$ Department of Physics, Faculty of Science, The University of Tokyo, Bunkyo-ku, Tokyo 113-0033, Japan
}

(Received 12 September 2021; accepted 19 October 2021; published 11 November 2021)

\begin{abstract}
Nambu-Goldstone bosons, or axions, may be ubiquitous. Some of the axions may have small masses and thus serve as mediators of long-range forces. In this paper, we study the force mediated by an extremely light axion, $\phi$, between the visible sector and the dark sector, where dark matter lives. Since nature does not preserve the $C P$ symmetry, the coupling between dark matter and $\phi$ is generically $C P$ violating. In this case, the induced force is extremely long range and behaves as an effective magnetic field. If the force acts on electrons or nucleons, the spins of them on Earth precess around a fixed direction toward the Galactic Center. This provides an experimental opportunity for $\phi$ with mass, $m_{\phi}$, and decay constant, $f_{\phi}$, satisfying $m_{\phi} \lesssim 10^{-25} \mathrm{eV}, f_{\phi} \lesssim 10^{14} \mathrm{GeV}$ if the daily modulation of the effective magnetic field signals in magnetometers is measured by using the coherent averaging method. The effective magnetic field induced by an axionic compact object, such as an axion domain wall, is also discussed.
\end{abstract}

DOI: 10.1103/PhysRevD.104.095010

\section{INTRODUCTION}

Despite the evidence of $C P$ violation, the strong sector has a very good $C P$-symmetric structure, which is unnatural and dubbed as a strong $C P$ problem. This problem may be solved by the existence of a QCD axion [1-4], which makes the QCD sector of the standard model (SM) settle into an almost $C P$-conserving vacuum.

Interestingly, string or M-theory may predict an axiverse [5-8], in which huge amounts of axions exist. The masses are generated by nonperturbative effects and thus spread over a wide range. Some of the string axions may be heavy, while some may be very light. Generic axions, or pseudoNambu-Goldstone bosons, have also been discussed widely [9-28], especially in the context of the dark matter (DM). Other than the dominant DM, some axion may form topological defects like cosmic strings or domain walls [29-31], and some may become the dark radiation [32-37]. Phenomenologically, a hint of the isotropic cosmic birefringence of cosmic microwave background polarization was reported [38]. The birefringence can be explained by the existence of (a) very light axion(s) or axionic

Published by the American Physical Society under the terms of the Creative Commons Attribution 4.0 International license. Further distribution of this work must maintain attribution to the author(s) and the published article's title, journal citation, and DOI. Funded by SCOAP . topological defects coupled to photons [31,38-41] (see also Refs. [42-50]). See Refs. [51-57] for reviews.

The axion can also play the role of long-range force contributing to the monopole-monopole, monopole-dipole, and dipole-dipole type interactions between the visible sector particles [58,59]. (See also Refs. [51,60,61].) To have a monopole source, we need a $C P$ violation between the axion and visible particles. Although the $C P$ feature of the SM highly suppresses the monopole coupling, one may get constraints comparable to the astronomical one $[62,63]$. Furthermore, the monopole-dipole interaction may provide strong evidence of the presence of the QCD axion in the ARIADNE experiment [64,65]. In general, the force becomes longer range if the axion mass is lighter.

In any case, a dark sector must exist to explain the missing mass of the Universe. Here we notice that, unlike the unnatural QCD sector, the dark sector may be generically $C P$ violating. For instance, as we show in Appendix A, in a dark QCD model, the dark nucleon provides a monopole force if we do not tune the strong $C P$ phase. With several axions as in the axiverse, the vacuum of the axion potential can be $C P$ violating, and then the axion DM emits the monopole force. Also, axionic topological defects generally emit the monopole force.

In this paper, therefore, we study the possibility that an ultralight axion plays the role of cosmically long-range force between the $C P$-violating dark sector and the visible sector particles. We call it a cosmic axion force. A dark sector particle or a compact object emits the cosmic axion 


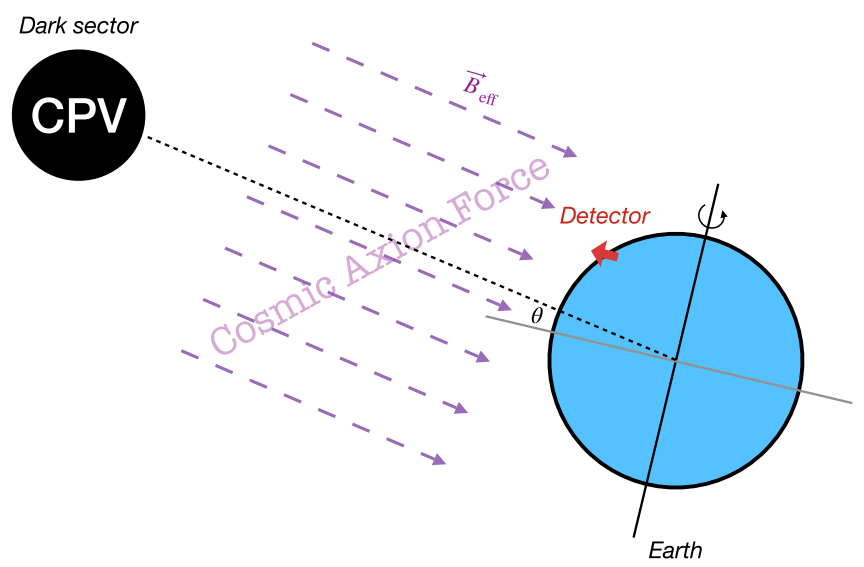

FIG. 1. A conceptual diagram of the detection for the cosmic axion force with a magnetometer. The long-range force emitted from a $C P$-violating dark sector, e.g., DM or compact objects (black), behaves like a constant magnetic field (purple dashed lines) that cannot be shielded as an ordinary magnetic field. Since the Earth (blue) is rotating along its axis, signals from the cosmic axion force measured by a local detector (red) have a daily modulation depending on the declination angle $\theta$.

force with a "monopole potential" $\propto 1 / r$. We point out that via the monopole-dipole interaction the cosmic axion force behaves as an effective magnetic field, which induces the spin precessions of axion-coupled SM fermions all over the Earth around a fixed direction (see Fig. 1). This axioninduced magnetic field remains intact when the ordinary magnetic fields are shielded. Such precession can be identified by carefully measuring the daily modulation of the effective magnetic field by magnetometers with a high enough sensitivity.

Magnetometers are popularly used in direct detections of the axion DM in the experiments of ABRACADABRA (Lumped Element) [66,67] and CASPEr [68-72] (see also Refs. [73,74]). In these cases, the induced magnetic field is along the direction of the DM velocity, and the strength is time varying. In contrast, the magnetic field induced by the cosmic axion force is toward the direction of the denser place of the source, e.g., the Galactic Center for the DM, and the strength is almost constant in time and, therefore, distinguishable from the direct detection of the DM. Compact object can be searched for in the magnetometers of GNOME [75-77]. The induced effective magnetic field is also time varying.

This paper is organized as follows. In the next section, we give the theoretical background on the cosmic axion force emitted from DM and a compact object and estimate the effective magnetic field for the spin precession. In Sec. III, we discuss the experimental opportunity. The last section is devoted to the conclusions.

\section{CP-VIOLATING AXION AND AXION FORCE}

An axion, $\phi$ which can be seen as a pseudo-NambuGoldstone boson, is a $C P$-odd scalar. Thus, with $C P$ symmetry and the shift symmetry, under which, respectively, an axion transforms

$$
\phi \rightarrow-\phi \quad \text { and } \quad \phi \rightarrow \phi+\alpha,
$$

the $\phi$ couplings to particles are controlled. Here, $\alpha$ is a real arbitrary number. However, $C P$ is violated in nature. This means, in general, the axion couples to particles, such as those in the dark sector, via $C P$-violating interaction (see Appendix A for concrete examples).

The exception is the couplings to the SM fermions. The coupling, especially to the nucleon, is almost $C P$ symmetric since $C P$ symmetry miraculously exists in the QCD sector; the nucleon electric dipole moment (EDM) has not been observed. There are also severe constraints in the fifth force searches [78-80], which not only constrains the $C P$ violating coupling to a nucleon but also an electron.

In this section, we study the axion mediated longrange force between the $C P$-violating dark sector and $C P$-preserving SM sector (by neglecting the CabibboKobayashi-Maskawa-induced effect for simplicity unless otherwise stated). We emphasize that we will not take $\phi$ as the QCD axion throughout this paper, but we consider it as a generic axion particle that may arise from string/ M-theory. The potential (around the minimum) is assumed to be

$$
V \supset \frac{m_{\phi}^{2}}{2} \phi^{2}
$$

This term violates the shift symmetry Eq. (1). We write this term since we would like to clarify the viable range for $\phi$ mass in our scenario. As we will see with $m_{\phi} \rightarrow 0$, our mechanism also works.

\section{A. Cosmic axion force from $C P$-violating dark sector}

Let us estimate the axion force from a $C P$-violating source, $J(t, \vec{x})$, in the dark sector. The equation of motion of $\delta \phi=\phi-\langle\phi\rangle$ with $\langle\phi\rangle$ being the vacuum expectation value, in flat spacetime, is given by

$$
\left(\partial_{t}^{2}-\nabla^{2}\right) \delta \phi(t, \vec{x})=-m_{\phi}^{2} \delta \phi(t, \vec{x})-J(t, \vec{x}),
$$

where $m_{\phi}$ is the mass of $\phi$. We parametrize

$$
J=\epsilon C[t] \frac{\rho_{\mathrm{DS}}(\vec{x})}{f_{\phi}},
$$

where $\epsilon$ quantifies the $C P$ violation in the dark sector, $C[t]$ is a model-dependent coefficient, which we will take to be 1 for simplicity; $f_{\phi}$ is the decay constant of the axion; and $\rho_{\text {DS }}$ represents the energy density of a dark sector field in the current Universe. In Appendix A, we show that various sources in concrete models of the dark sector can be 
represented in this form. We also show the case when $C[t]$ is varying in time.

To solve the equation from a general source, we first calculate the potential from a point source:

$$
J^{\mathrm{ps}}(t, \vec{x})=\delta^{3}(\vec{x}) .
$$

We obtain the solution to Eq. (3) as

$$
\delta \phi^{\mathrm{ps}}(t, \vec{x})=\frac{1}{4 \pi r} \exp \left(-m_{\phi} r\right),
$$

where $r=|\vec{x}|$. As a result, the force from a general nonrelativistic, stationary, distribution can be given by the superposition of

$$
\delta \phi(t, \vec{x}) \approx \int d^{3} \vec{x}^{\prime} \frac{\epsilon \rho_{\mathrm{DS}}\left(x^{\prime}\right)}{f_{\phi}} \delta \phi^{\mathrm{ps}}\left(t,\left|\vec{x}-\overrightarrow{x^{\prime}}\right|\right) .
$$

The energy density of the dark sector, $\rho_{\text {DS }}$, has "charge" $\epsilon / f_{\phi}$ for the force. Note that, even if $\epsilon$ is nonvanishing, the force $\vec{\partial} \delta \phi$ would be vanishing if $\rho_{\mathrm{DS}}$ were spatially homogeneous. However, as we will see, the dark sector density is generally spatially inhomogeneous.

\section{B. Cosmic axion force acting on $\boldsymbol{C P}$-preserving SM sector}

We can discuss the phenomenon of the long-range force mediated by $\phi$. From the aforementioned reasons, we assume that the axion $\phi$ has shift and $C P$-symmetric interactions to SM fermions $\psi$. The lowest dimension term is given as

$$
\mathcal{L}=\frac{c_{\psi} \partial_{\mu} \phi}{f_{\phi}} \bar{\psi} \gamma_{5} \gamma^{\mu} \psi
$$

where $c_{\psi}$ is a dimensionless constant. If there is such a term coupling to quarks, one obtains the couplings to the nucleons below the QCD scale

$$
\rightarrow \frac{c_{N} \partial_{\mu} \phi}{f_{\phi}} \bar{N} \gamma_{5} \gamma^{\mu} N
$$

where $c_{N}$ is a constant which is related with $c_{\psi}$ for quarks and $N=p, n$ is a nucleon. We emphasize that $\phi$ is not a QCD axion. Since interaction (8) itself is completely shift symmetric, the QCD instanton does not generate the potential of $\phi$. In other words, the shift symmetry is anomaly free to the color gauge group [53,81-88].

At the nonrelativistic limit of the fermion, one obtains the interacting Hamiltonian as

$$
H \simeq-\frac{c_{i}}{f_{\phi}} \vec{\partial} \delta \phi \cdot \vec{\sigma}_{i}
$$

Here, $i=e, \mu, n, p$, etc. We can make a proper Lorentz transformation from/to this basis to get the Hamiltonian in the relativistic limit. ${ }^{1}$

The Hamiltonian resembles the one for magnetic moment interaction, $\mu_{i} \vec{B} \vec{\sigma}_{i}$. One can identify the "magnetic field" of

$$
\vec{B}_{\text {eff }} \equiv \frac{c_{i} \vec{\partial} \delta \phi}{\left(\mu_{i} f_{\phi}\right)}
$$

coupled to the "magnetic moment," $\mu_{i} \vec{\sigma}$. The neutron $(i=n)$ has the value of $\mu_{n} \approx-1.9 \mu_{0}$, and a proton $(i=p)$ has $\mu_{p} \approx 2.8 \mu_{0}$, where $\mu_{0} \equiv e / 2 m_{N} \approx 0.1 e \cdot \mathrm{fm}$ is the nuclear magneton. For the charged lepton $i=e, \mu, \tau$, $\mu_{i} \approx e / 2 m_{i}$. Because of the magnetic field, the spin of the fermion precesses. The precession frequencies are given as

$$
\begin{gathered}
f_{\text {electron }}=2.4 \text { day }^{-1} \times \frac{\left|\vec{B}_{\text {eff }}\right|}{f T} \\
f_{\text {proton }}=1.3 \text { year }^{-1} \times \frac{\left|\vec{B}_{\text {eff }}\right|}{f T}
\end{gathered}
$$

for the electron and proton, respectively.

\section{Cosmic axion force from DM}

Let us give some concrete examples for the cosmic axion forces. A most important source perhaps is the DM. Because of the primordial density perturbation and structure formation, the DM must be spatially inhomogeneous. Alternatively, we may also have compact objects formed by DM [25,90] or topological defects such as domain walls [29-31], which will be our later topic.

We divide the energy density of the DM, $\rho_{\mathrm{DS}}$, into two parts,

$$
\rho_{\mathrm{DS}}=\rho_{\text {galactic }}+\rho_{\mathrm{extra}}
$$

where $\rho_{\text {galactic }}\left(\rho_{\text {extra }}\right)$ is the energy density contribution from our Galaxy by assuming some standard distributions (extra Galactic component).

\footnotetext{
${ }^{1}$ Since $\delta \phi$ is time independent, boosting a fermion does not enhance the spin precession frequency (it is enhanced in the rest frame by a Lorentz factor due to the Lorentz contraction, but it cancels out in the Laboratory frame due to the time dilation). This is different from the fermion precession in an oscillating axion DM background. In this case, the spin precision is proportional to the velocity, and thus the precession frequency is enhanced by a Lorentz boost [89]. On the other hand, a deuteronlike particle has a negative anomalous magnetic moment. In a storage-ring experiment of the particle, the spin can be frozen in the laboratory frame. It may be a good experimental tool for searching for the cosmic axion force.
} 
Let us adopt the Navarro-Frenk-White (NFW) DM profile for $\rho_{\text {galactic }}[91,92]$. One can calculate the force contribution from

$$
\rho_{\text {galactic }}=\rho_{\mathrm{NFW}}=\frac{\rho_{s} r_{s}}{r}\left(1+\frac{r}{r_{s}}\right)^{-2}
$$

where $\rho_{s} \approx 0.184 \mathrm{GeV} / \mathrm{cm}^{3}, r_{s} \approx 24.43 \mathrm{kpc}$, and the position of the Sun is at $r \approx r_{\odot} \approx 8.33 \mathrm{kpc}$. Then, one can calculate the potential from Eq. (7) and obtain the cosmic axion force by taking the derivative. This contribution gives $B_{\text {eff }}$ toward or opposes to the Galactic Center depending on the sign of $c_{i} \epsilon$. This is because the DM distributes spherically around the Galactic Center in the NFW profile. With the other DM profile for $\rho_{\text {galactic }}$, the resulting cosmic axion force does not change much. For instance, if we adopt the Burkert profile [93,94] (see also Ref. [95]), the force will differ at most by $\mathcal{O}(10 \%)$ in the range $m_{\phi} \lesssim 1 / r_{\odot}$ with using the parameters in Ref. [92].

In most of the space within the horizon, $\rho_{\text {extra }}$ should be $\rho_{\text {crit }} \times \Omega_{\mathrm{DM}}$, where the critical density of the Universe $\rho_{\text {crit }} \approx 1.1 \times 10^{-5} h^{2} \mathrm{GeV} / \mathrm{cm}^{3}$ and $\Omega_{\mathrm{DM}} h^{2} \approx 0.12$ [96], with $h \approx 0.67$ being the reduced Hubble parameter. An inhomogeneous distribution must exist due to the primordial density perturbation of $\mathcal{O}\left(10^{-3}\right) \%$ from inflation [96]. We parametrize the inhomogeneity of $\rho_{\text {extra }}$ as

$$
\begin{aligned}
\vec{\xi} \equiv & \left(\rho_{\text {crit }} \Omega_{\mathrm{DM}} \frac{1}{3 m_{\phi}}\right)^{-1} \int d^{3} \overrightarrow{x^{\prime}} \rho_{\mathrm{extra}}\left(\overrightarrow{x^{\prime}}\right) \\
& \times \frac{\vec{x}-\overrightarrow{x^{\prime}}}{\left|\vec{x}-\overrightarrow{x^{\prime}}\right|^{3}} \exp \left(-m_{\phi}\left|\vec{x}-\overrightarrow{x^{\prime}}\right|\right) .
\end{aligned}
$$

If the dark sector distribution is spatially inhomogeneous, $\xi \neq 0$. We turn on this contribution if $r>r_{s}$. If the $\rho_{\text {extra }}$ contribution is dominant, we obtain an effective magnetic field of $\mu_{i} \vec{B}_{\text {eff }}=\epsilon \vec{\xi} \frac{c_{i} \rho_{\text {crit }} \times \Omega_{\mathrm{DM}}}{f_{\phi}^{2} m_{\phi}}$, which should increase if we decrease $m_{\phi}$ by fixing $f_{\phi}$. However, as we will see soon, this component is subdominant when $m_{\phi}$ is greater than the Hubble constant.

The cosmic axion force induces the spin precession of the SM fermions that couple to the axion. The effective magnetic fields are given in Fig. 2 with $\xi=10^{-5}$ for the nucleon and electron precessions in the upper and lower panels, respectively, with $c_{p}=1$ and $c_{e}=1 . \epsilon=1$ is fixed. Red dotted contours represent the possible sensitivity reaches of magnetometers (see Sec. III). The shaded region in the bottom may be excluded by astrophysical bounds (see the following). With general $\epsilon, c_{i}$, the vertical axis can be regarded as $\log _{10}\left(\sqrt{\left(g_{a \psi_{i} \psi_{i}}^{(p)} g_{a \mathrm{DMDM}}^{(s)}\right)^{-1} m_{\psi_{i}} m_{\mathrm{DM}}} / \mathrm{GeV}\right)$ with $g_{a \psi_{i} \psi_{i}}^{(p)}=c_{\psi_{i}} m_{\psi_{i}} / f_{\phi}$ and $g_{a \mathrm{DMDM}}^{(s)}=\epsilon m_{\mathrm{DM}} / f_{\phi}$ (see also Appendix A for $g_{a \mathrm{DMDM}}^{(s)}$ ). When $1 / m_{\phi}$ is much larger
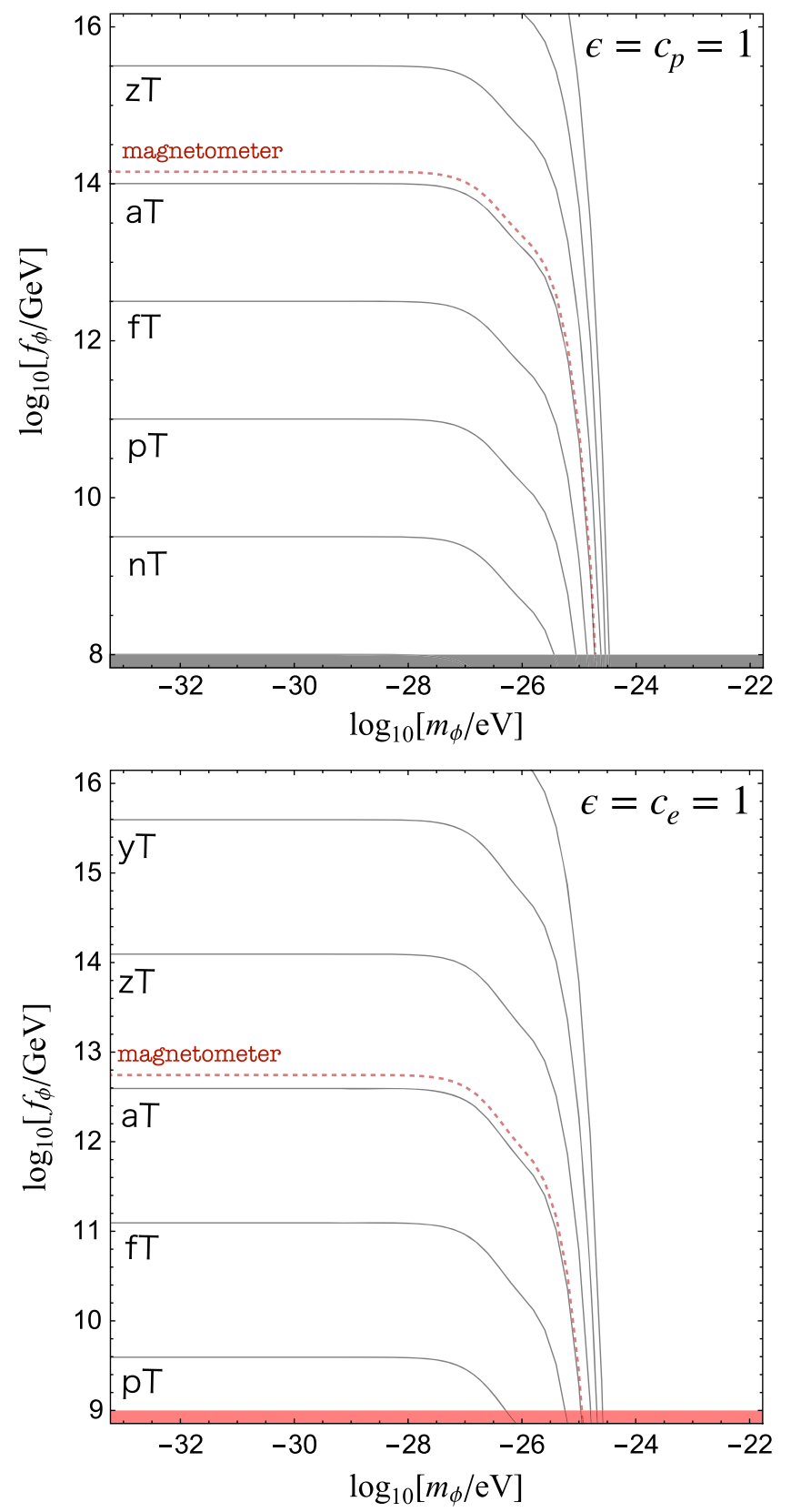

FIG. 2. Contours of the effective magnetic field, $\left|\vec{B}_{\text {eff }}\right|[\mathrm{T}]$, for proton (upper panel) and electron (lower panel) from the cosmic axion force induced by the DM with $c_{p}=1$ and $c_{e}=1$, respectively. In both figures, we take $\epsilon=1$ and assume the NFW DM profile. The direction is toward or opposes to the Galactic Center. The lower colored region may be excluded by astrophysical bounds. The red dotted curve represents $\simeq 0.5 \mathrm{aT}$, the sensitivity reaches for the magnetometers estimated in the next section.

than $r_{\odot}$, the induced magnetic field is almost constant. This is because the force from $r \gg r_{\odot}$ cancels out. This effect represents that the force is dominantly from the DM around the Galactic Center. When $1 / m_{\phi}$ is much larger than the size of our Galaxy, on the other hand, the force from the 
density perturbation should become more important in principle. However, from the numerical estimation, we found that the extra-Galactic component is subdominant in the mass range shown in the figure. When $1 / m_{\phi}$ is larger than the range shown in the figure, i.e., larger than the horizon size, the cosmic axion force from out of the Hubble horizon should not reach us. Therefore, we can conclude that the robust prediction of this scenario is the direction of the effective magnetic field, which is toward or opposes to the Galactic Center. ${ }^{2}$

If the dark sector particle, on the other hand, forms a compact object, the force direction might be biased to the object. We can use $\xi \gg 10^{-5}$ to estimate the size of the magnetic field for this effect. The formation of a compact object, the position, and the typical $\xi$ are model dependent. We will discuss the possibility of the cosmic axion force from an axion domain wall in Sec. II D.

\section{Constraints on the DM scenario}

The axion is extremely light, and it couples to the SM fermions. Such an axion may be constrained from astrophysics and cosmology. Since the energy density or the potential height, $\rho_{\phi} \ll f_{\phi}^{2} m_{\phi}^{2}$, is extremely small in the viable region $\left(f_{\phi} \lesssim 10^{14} \mathrm{GeV}\right)$, the constraints for the overproduction of $\phi$ as DM or dark energy [97] are negligible. However, there is an important constraint: the stellar cooling. If $\phi$ couples to nucleon (electron), $f_{\phi} \gtrsim$ $10^{8} \mathrm{GeV}\left(f_{\phi} \gtrsim 10^{9} \mathrm{GeV}\right)$ is required from the cooling constraints of SN1987A [98-101] (red giant stars $[102,103])$. With $f_{\phi} \lesssim 10^{8} \mathrm{GeV}$, one should also care axion monopole force induced by the nucleon in the $\mathrm{QCD}$ sector via $C P$ violation $[58,59]$. If the strong $C P$ phase, $\theta_{\mathrm{CP}}$, is nonvanishing, an axion-nucleon Yukawa coupling is induced $g_{a N N} \sim \theta_{\mathrm{CP}} f_{\pi} / f_{a}$ with $f_{\pi} \sim 130 \mathrm{MeV}$ being the pion decay constant. In fact, the strong $C P$ phase should not be completely vanishing and should satisfy $10^{-17}-10^{-16} \lesssim\left|\theta_{\mathrm{CP}}\right| \lesssim 10^{-10}$. Here, the upper bound is from the nonobservation of the neutron EDM, and the lower bound comes from the electroweak contribution (by assuming a QCD axion) [104]. Even if we introduce the $\mathrm{QCD}$ axion to solve the strong $C P$ problem, a $C P$-violating weak interaction drives the minimum of the QCD axion slightly away from the $C P$-conserving place. By taking into account the $C P$-violating effect, the nucleon also induces a monopole potential, which is constrained by the aforementioned test of monopole-monopole interaction [78] and monopole-dipole interaction [105] between visible particles (see also Refs. $[51,60,61])$. We get the constraint $f_{\phi} \gtrsim 10^{7} \mathrm{GeV}$ if $\theta_{\mathrm{CP}}=10^{-17}$ for $m_{\phi} \lesssim 10^{-15} \mathrm{eV}$. We find

\footnotetext{
${ }^{2}$ We have used $\xi$ parameter to take account the extra-Galactic contribution. In addition, we have checked that the axion force from Virgo cluster is smaller than the Galactic component by $2-3$ orders of magnitude when the axion force is reachable.
}

that the reach of $f_{\phi} \sim 10^{14} \mathrm{GeV}$ for our monopole-dipole interaction between dark and visible sectors can be many orders of magnitude beyond those set by the astrophysical and ordinary long-range force constraints.

The reason that the cosmic axion force from DM can be very strong is that the force is from a constructive superposition over a cosmological scale. This is similar to the fact that we feel gravity despite it being extremely weaker than the electromagnetic force. The reason that the interaction between dark and visible sectors is less constrained than the one between visible sectors is the difficulty of measuring the dark sector interaction. Indeed, the constraint on DM monopole-monopole interaction is even weaker [106]. For instance, the asymmetric DM from dark baryon (see Appendix A), which may have mass around $m_{N^{\prime}}=$ $\mathcal{O}(1) \mathrm{GeV}$, should satisfy $f_{\phi} \gtrsim 10^{-2} m_{N^{\prime}}$.

\section{Cosmic axion force from domain wall}

A simple example for a compact object or a topological defect is a domain wall. There are various mechanisms to form the axion domain wall [29-31]. The interesting point for our scenario is that the interaction between an axion and a axion domain wall is generically $C P$ violating (see the Appendix A). If the axion domain wall follows the so-called scaling solution, we expect $\mathcal{O}(1)$ domain walls within a Hubble horizon. To evade the domain wall problem, the tension of the domain wall should satisfy $[107,108]$

$$
\sigma_{\mathrm{DW}} \lesssim(1 \mathrm{MeV})^{3}
$$

If the axion domain wall couples to another axion, $\phi$, the long-range force is emitted from the wall. The limited amount of the domain walls implies that the force is difficult to cancel out unless we tune the position and direction of several domain walls. In the following, we consider for simplicity that there is only a single domain wall inside our Hubble horizon and stretch over perpendicular to the position vector, $\vec{r}_{\mathrm{DW}}$.

The potential from a nonrelativistic domain wall can be calculated as

$$
\delta \phi(t, \vec{x}) \approx \int d^{2} \vec{x}_{\perp} \frac{\epsilon \sigma_{\mathrm{DW}}}{f_{\phi}} \delta \phi^{\mathrm{ps}}\left(t,\left|\vec{x}-\overrightarrow{x^{\prime}}\right|\right)
$$

where the integral is performed on the surface of the domain wall. Then, we obtain, analytically,

$$
\delta \phi(t, \vec{x})=\epsilon \frac{\sigma_{\mathrm{DW}}}{f_{\phi}} \frac{e^{-m_{\phi}\left|\vec{r}_{\mathrm{DW}}\right|}}{2 m_{\phi}} .
$$

The force is perpendicular to the wall and behaves as an effective magnetic field, e.g., for a proton as 
$\left|\vec{B}_{\text {eff }}\right|=3 \mathrm{aT} \times \gamma_{\mathrm{DW}} \epsilon c_{p} e^{-\left|\vec{r}_{\mathrm{DW}}\right| m_{\phi}}\left(\frac{10^{12} \mathrm{GeV}}{f_{\phi}}\right)^{2} \frac{\sigma_{\mathrm{DW}}}{(1 \mathrm{MeV})^{3}}$,

where $\gamma_{\mathrm{DW}} \equiv 1 / \sqrt{1-\left(\dot{\vec{r}}_{\mathrm{DW}}\right)^{2}}$ is the Lorentz factor for the domain wall motion, which takes into account the Lorentz contraction and may enhance the magnetic field. This is exponentially suppressed if $\left|\vec{r}_{\mathrm{DW}}\right|$ is larger than $1 / m_{\phi}$. For the cosmic axion force mediated by a lighter axion than $1 /\left|\vec{r}_{\mathrm{DW}}\right|$, the effective magnetic field does not depend much on the distance to the domain wall. Therefore, the cosmic axion force can be mediated to Earth from an extremely distant domain wall.

We may wonder if the axion, $a$, forming the domain wall can also mediate the force. Indeed, there is a force mediated by $a$. That is nothing but the gradient of the domain wall configuration. The corresponding magnetic field is now being searched for in the GNOME experiment [75-77]. The experimental advantage of introducing another light axion is that the force range, $1 / m_{\phi}$, can be much longer than the wall size $1 / m_{a}$ with $m_{\phi} \ll m_{a}$. Then, the probability for the interaction with the detector on Earth is extremely enhanced. The disadvantage may be that the magnetic field is almost a constant value unless $\left|\vec{r}_{\mathrm{DW}}\right| \sim$ $1 / m_{\phi}$ and the domain wall is moving.

\section{MEASUREMENT OF COSMIC AXION FORCE}

So far, we have discussed some theoretical points of the cosmic axion force, which behaves as the effective magnetic field and induces the spin precession of the SM fermions.

This magnetic field features the following properties:

(i) The effective magnetic field is kept intact even if we shield all the ordinary magnetic fields away (see also the discussions in Sec. III C for electron coupling).

(ii) The effective magnetic field is toward a fixed direction, as the Galactic Center, everywhere on Earth.

(iii) The effective magnetic field is almost constant within an experimental timescale say $>1$ day.

There are several methods to test the fictitious magnetic field using highly sensitive methods such as the optical magnetometer, SQUID magnetometer, or nuclear magnetic resonance. The optical magnetometer, especially the spinexchange relaxation free (SERF) comagnetometer, is one of the promising methods to test the cosmic axion force acting on the polarized nuclei due to the insensitivity of the detector to the magnetic field drift and gradient. For instance, the $\mathrm{K}-{ }^{3} \mathrm{He}$ comagnetometer [109] is composed of two polarized spin elements, $\mathrm{K}$ (electron) and ${ }^{3} \mathrm{He}$ (nuclei). The polarized ${ }^{3} \mathrm{He}$ suppresses the ambient ordinary magnetic fields and gradients to the $\mathrm{K}$ spin. The ${ }^{3} \mathrm{He}$ spin cannot suppress the fictitious magnetic field induced by the axion force. Therefore, the net (effective) magnetic field acting on the $\mathrm{K}$ spin is nonzero, and thus the spin rotates. From an optical method, we can measure the slowly varying magnetic field via the $\mathrm{K}$ spin rotation. Because of the stability of the spin system, the $\mathrm{K}-{ }^{3} \mathrm{He}$ comagnetometer works at frequency $\lesssim 5 \times 10^{-10} \mathrm{~Hz}$ [110], which includes the region of our interest. When the axion couples to quarks, the induced effective magnetic field on the ${ }^{3} \mathrm{He}$ is of the order of the proton one. Thus, we can use the upper panel of Fig. 2 to estimate this case.

\section{A. Magnetometers and daily modulation}

A magnetically shielded atomic magnetometer could be a detector for the pseudomagnetic field, $\vec{B}_{\text {eff }}$. It effectively screens conventional magnetic fields for a whole day, and the axionic monopole signals can be accumulated. However, usually, an atomic magnetometer has directional sensitivity, and this direction will have a precession unless it is orthogonal to the axial tilt direction. Here, we consider the detector directing along the celestial equator, for simplicity, to reduce precession error.

When the magnetometer is operating, the axionic monopole gives a constant signal for a short period. By taking into account the Earth's rotation, the signals have daily modulation. Figure 1 illustrates a conceptual diagram of the possible monopole (black) search using the atomic magnetometer as a detector (red) in the Earth (blue). The angle between the monopole position vector from the detector and its sensitive direction is $\theta$. Thus, the daily modulation depends on $\theta$. Roughly speaking, the signal amplitude is reduced by a factor of $\cos (\theta)$. Then, the daily modulation peak-to-peak amplitude will be

$$
\delta B \approx 2\left|\vec{B}_{\mathrm{eff}}\right| \cos \theta,
$$

where the cosine term gives $\cos \theta \geq 0.1$ for $\theta \leq 84.3^{\circ}$. For practical case, one has

$$
\delta B \geq 0.2\left|\vec{B}_{\text {eff }}\right| .
$$

In the following, we estimate the sensitivity, by taking care of the daily modulation by taking $\cos \theta=\mathcal{O}(1)$. We note that, since we know the direction of the Galactic Center (the Galactic longitude and latitude $\sim 0$ ), we can optimize the direction of the detector when we measure the force from DM.

\section{B. Sensitivity estimation}

There are two ways to average data depending on the experiment conditions: incoherent averaging and coherent averaging (see Appendix B). Although incoherent averaging conserves energy during the averaging process, coherent averaging reduces the energy components of noise, 
resulting in higher signal-to-noise ratio (SNR) for the same average number. Coherent averaging can be utilized if the signal has coherence during the measurement time, or if the phase of the signal is known. The cosmic axion force is supposed to be constant during the measurement time. But the magnetic field induced by the cosmic axion force oscillates, for example, with the rotation of the Earth (or rotation of the detector). In this case, the phase of the cosmic axion-induced magnetic field can be recorded. The measured data can be coherently averaged, and the SNR is expressed as

$$
\mathrm{SNR}=\frac{B_{\text {sig }}}{B_{\text {noise }}} \sqrt{2 N},
$$

where $B_{\text {sig }}$ is the strength of the cosmic axion force-induced magnetic field in the frequency domain, $B_{\text {noise }}$ is the magnetic field noise fluctuation in the frequency domain, $N$ is the number of experiments that perform a coherent averaging, and the $\sqrt{2}$ factor is attributed to the experimental characteristics that utilize only one component of the frequency component of noise using the phase information. The noise fluctuation $B_{\text {noise }}$ can be written with magnetic field spectral density $\left(\delta B_{n}\right)$ and the resolution bandwidth $b$ :

$$
B_{\text {noise }}=\delta B_{n} \sqrt{b} .
$$

For example, the nucleon-electron comagnetometer has sensitivity order of $1 \mathrm{fT} / \sqrt{\mathrm{Hz}}$ range in the low-frequency region less than $1 \mu \mathrm{Hz}$ [109-111]. The target sensitivity is set with (to be) a target SNR of 5, averaging over 300 sidereal days with a resolution bandwidth of $20 \mu \mathrm{Hz}$. The detectable signal, then, can be estimated as

$B_{\text {sig }} \approx 0.67 \mathrm{aT}\left[\frac{\mathrm{SNR}}{5}\right]\left[\frac{300}{N}\right]^{0.5}\left[\frac{\delta B_{n}}{1 \mathrm{fT} / \mathrm{Hz}}\right]\left[\frac{b}{20 \mu \mathrm{Hz}}\right]^{0.5} \frac{1}{\sqrt{2}}$.

\section{Magnetic shielding and force direction measurement}

Most atomic magnetometers use either alkali metals or noble gases. Those magnetometers have two steps (if one omits optical pumping): first, spin precession motion with angular frequency $\omega$ under magnetic field $B$,

$$
\omega=\gamma B,
$$

where $\gamma$ is the gyromagnetic ratio of an optical medium, and the second step is probing a polarization by dichroism or birefringence.

We have started with an atomic magnetometer having an exotic electron spin coupling to a light axion. However, since the axion force also acts on the electron in a ferro-/ferrimagnetic shielding, this electron coupling could be screened [112]. Since they are conventionally used in atomic magnetometers, the effective magnetic field seen by an electron will be suppressed by $\mathcal{O}(100)$ if this effect fully occurs. However, we argue that this problem may be avoided if the effective magnetic field is in sub-femto Tesla range. The timescale of the induced motion of an electron spin is longer than a day [see Eq. (12)]; i.e., the induced magnetic field for the shielding is in the timeaveraged force direction in the laboratory frame. However, the (daily modulating) spin precession may be around a different direction than the original force direction due to the shielding. In this case, we may measure the force direction with several detectors at different places like in GNOME. An alternative simple possibility to avoid this problem is to rotate the shield much faster than $f_{\text {electron }}$ while keeping the magnetometer intact.

Instead, nucleon coupling is not affected by the magnetic shielding, and we do not need to care about this issue. From the daily modulation of the nucleon spin motion, we can measure the force direction. It will be smoking-gun evidence of our scenario if the force direction is toward the Galactic Center.

\section{CONCLUSIONS}

We have studied a very light axion as a mediator of a long-range force between the dark sector and visible sector. The key assumption is that the dark sector, from which the force originates, has $C P$ violation. Even if the dark sector component is extremely far away, it can affect the laboratory detector on Earth via the long-range force. The force behaves as a magnetic field, which induces the spin precessions of nucleons or leptons. The precession is around a fixed direction and is kept even with an ordinary magnetic field shielding. Such precessions can be detected in magnetometers via the daily modulation of signals. The constant effective magnetic field toward the Galactic Center is a smoking-gun prediction of the cosmic axion force from the dark matter.

Let us mention a few extensions of our proposal. First of all, a similar scenario can be obtained if the dark sector is charged under a dark Abelian gauge group, whose gauge field couples to the visible particles via electric dipole moment operators. Another interesting possibility is that the axion $\phi$ forms nontrivial distribution around an ordinary star like in Ref. [113,114], which discusses finite density corrections for the QCD axion potential in a neutron star. In our case, $\phi$ does not get the potential from QCD instanton, and this effect is neglected. However, the Peccei-Quinn symmetry can be restored inside a star if the Peccei-Quinn field for the axion is light enough compared to the finite density effect inside a star. The Peccei-Quinn field and matter coupling may be caused by the mixing between the Peccei-Quinn field and the Higgs field. Then, the axion field may obtain a nontrivial distribution around the star, and thus the gradient of $\phi$ behaves as the cosmic axion 
force. In this case, the force direction may be almost toward or opposed to Sun, especially when $1 / m_{\phi}$ is not much larger than the distance between the Earth and Sun.

\section{ACKNOWLEDGMENTS}

W. Y. would like to thank the members at Center for Axion and Precision Physics Research for kind hospitality when this work was initiated and for the warm help when he was in difficulty due to COVID-19. We thank Paolo Salucci and Fuminobu Takahashi for useful comments. The work of Y.C.S. and Y.K.S. is supported by the Institute for Basic Science under Grant No. IBS-R017-D12021-a00. W. Y. was supported by JSPS KAKENHI Grants No. 16H06490, No. 19H05810, No. 20H05851, and No. 21K20364.

\section{APPENDIX A: DARK SECTOR MODELS WITH $C P$-VIOLATING AXION COUPLING}

Let us explain that the axion coupling to the dark sector may easily violate $C P$. To restrict ourselves, let us consider that the theory has $C P$ symmetry at high-energy scales (at the perturbative level).

\section{Dark QCD with a strong $C P$ phase}

For instance, we can consider a QCD-like model (we call it dark QCD) with a nonvanishing strong $C P$ phase coupled to the axion with (1),

$$
\mathcal{L} \supset-\theta_{\mathrm{CP}}^{\prime} \tilde{G}^{\prime} G^{\prime}-\frac{c_{\psi^{\prime}} \partial_{\mu} \phi}{f_{\phi}}\left(\overline{u^{\prime}} \gamma_{5} \gamma^{\mu} u^{\prime}+\bar{d}^{\prime} \gamma_{5} \gamma^{\mu} d^{\prime}\right),
$$

where we have neglected to write down the kinetic (and mass) terms. Again, we notice that the axion does not solve the strong $C P$ problem for this model since the Lagrangian is shift symmetric under $\phi \rightarrow \phi+\alpha$ and $\phi$ never has a potential to eliminate $\theta_{\mathrm{CP}}^{\prime}$. Here, we have assumed that the dark quarks have two flavors, and the axion couples to them universally. This is quite similar to the ordinary two-flavor QCD except for the $C P$ phase and the axion couplings. Thus, we expect confinement for large enough gauge coupling. Then, the dark nucleon, $N^{\prime}$, will have a $C P$ violating Yukawa interaction, $\mathcal{L}_{\text {eff }} \supset-g_{\phi N^{\prime} N^{\prime}} \phi \bar{N}^{\prime} N^{\prime}$, with the coupling satisfying $[58,59]$

$$
g_{\phi N^{\prime} N^{\prime}}=\frac{\theta_{\mathrm{CP}}^{\prime} c_{\psi^{\prime}}}{f_{\phi}} \frac{m_{u^{\prime}} m_{d^{\prime}}}{\left(m_{u^{\prime}}+m_{d^{\prime}}\right)}\left\langle N^{\prime}\left|\bar{u}^{\prime} u^{\prime}+\bar{d}^{\prime} d^{\prime}\right| N^{\prime}\right\rangle .
$$

If $m_{u^{\prime}} \sim m_{d^{\prime}} \sim\left\langle N^{\prime}\left|\bar{u}^{\prime} u^{\prime}+\bar{d}^{\prime} d^{\prime}\right| N^{\prime}\right\rangle \sim m_{N^{\prime}}$, with $m_{N^{\prime}}$ being the mass of the dark nucleon, we obtain the $C P$-violating coupling of order

$$
g_{\phi N^{\prime} N^{\prime}} \sim c_{\psi^{\prime}} \theta_{\mathrm{CP}}^{\prime} \frac{m_{N^{\prime}}}{f_{\phi}} .
$$

For comparison, we mention that in the ordinary QCD, due to the fine-tuning of the strong $C P$ phase, this term is small. However, if $\theta_{\mathrm{CP}}^{\prime}$ is not finely tuned, we have $C P$-violating coupling between axion and the nucleon. When the dark nucleon composes the dominant DM, we get $\varepsilon \sim \theta_{\mathrm{CP}} c_{\psi}$, and thus $\varepsilon=\mathcal{O}(1)$ if there is no tuning. The dark nucleon could also be the asymmetric DM, in which case the mass is $m_{N}=\mathcal{O}(1) \mathrm{GeV}$.

\section{Spontaneous $\boldsymbol{C P}$ breaking in axiverse}

In axiverse, many axions, $\phi_{i}$, have a potential generated by a nonperturbative effect,

$$
V=V\left(\phi_{i} / f_{i}\right),
$$

where $f_{i}$ is the decay constant. The axions enjoy discrete shift symmetry

$$
\phi_{i} / f_{i} \rightarrow \phi_{i} / f_{i}+2 \pi,
$$

under which the potential is invariant,

$$
V\left(\phi_{i} / f_{i}\right)=V\left(\phi_{i} / f_{i}+2 \pi\right) .
$$

The periodicity implies that the potential can be given in the form

$$
V=-\Lambda^{4} \sum_{n_{j}} \kappa_{j} \cos \left(\sum_{i} n_{i}^{j} \frac{\phi_{i}}{f_{i}}+\theta_{j}\right)
$$

where $n_{i}^{j}$ are integers. (We omit the constant term to cancel the vacuum energy today.) Immediately, we find that there are $C P$ phases, $\theta_{j}$. Therefore, there could be $C P$ violation in general.

Even if we take the " $C P$-symmetric" limit, $\theta_{j}=0$, the $C P$ symmetry can be spontaneously broken. For simplicity, we consider a two-axion model with potential given by

$$
\begin{aligned}
V= & -\Lambda^{4}\left(\kappa_{1} \cos \left(n_{a} \frac{a}{f_{a}}\right)+\kappa_{2} \cos \left(\frac{\phi}{f_{\phi}}+\frac{a}{f_{a}}\right)\right. \\
& \left.+\kappa_{3} \cos \left(n_{\phi} \frac{\phi}{f_{\phi}}\right)\right)
\end{aligned}
$$

with $\kappa_{1} \gtrsim \kappa_{2}, \kappa_{3}, n_{a}>1$; then, we can integrate out $a$, which has local minima $\langle a\rangle / f_{a} \approx 0,2 \pi / n_{a}, 4 \pi / n_{a} \cdots$ $\left(n_{a}-1\right) 2 \pi / n_{a}$. The mass of $a$ is

$$
m_{a}^{2} \sim \kappa_{1} \frac{n_{a}^{2} \Lambda^{4}}{f_{a}^{2}} .
$$

Except for the first minimum, we obtain a nonvanishing $C P$ phase from the spontaneous symmetry breaking. This appears in the low-energy theory as 
$V_{\mathrm{eff}} \simeq-\Lambda^{4}\left(\kappa_{2} \cos \left(\theta+\frac{\phi}{f_{\phi}}\right)+\kappa_{3} \cos \left(n \frac{\phi}{f_{\phi}}\right)\right)$

where $\theta \equiv\langle a\rangle / f_{a} \cdot{ }^{3}$ Let us expand the potential around the minimum of $\phi$. We obtain

$$
V_{\mathrm{eff}} \simeq \frac{m_{\phi}^{2}}{2} \delta \phi^{2}+\frac{A_{\phi}}{3 !} \delta \phi^{3}+\cdots
$$

Here, $\delta \phi \equiv \phi-\langle\phi\rangle$, with $\langle\phi\rangle / f_{\phi} \simeq-\theta \kappa_{2} /\left(\kappa_{2}+n^{2} \kappa_{3}\right)$,

$$
m_{\phi}^{2} \simeq\left(\kappa_{2}+n^{2} \kappa_{3}\right) \frac{\Lambda^{4}}{f_{\phi}^{2}},
$$

and

$A_{\phi} \simeq \theta \frac{\kappa_{2} \kappa_{3} n^{2}}{\kappa_{2}+\kappa_{3} n^{2}}\left(1-n^{2}\right) \frac{\Lambda^{4}}{f_{\phi}^{3}}+\mathcal{O}\left(\theta^{3}\right) \equiv \epsilon_{\phi} \frac{m_{\phi}^{2}}{f_{\phi}}$

which is nonvanishing if $\theta \neq 0$. This term is obviously $C P$ violating. This will give a source term for the long-range force discussed in the next subsection.

Let us come back to the original potential Eq. (A8); by defining $\delta a \equiv a-\langle a\rangle$, we obtain the interacting term from the second cosine term as

$$
V \supset \frac{A_{a}}{2} \delta a^{2} \delta \phi
$$

where

$$
A_{a} \simeq \theta \frac{\kappa_{2} \kappa_{3} n^{2}}{\kappa_{2}+\kappa_{3} n^{2}} \frac{\Lambda^{4}}{f_{a}^{2} f_{\phi}}+\mathcal{O}\left(\theta^{3}\right) \equiv \epsilon_{a} \frac{m_{a}^{2}}{f_{\phi}} .
$$

Consequently, other than the self-cubic-interaction, the $C P$ breaking also induces the cubic interaction between heavier, $a$, and lighter, $\phi$, axions. We mention that $a$ can be the QCD axion if it is anomalously coupled to the gluons. In this case, we may identify $\kappa_{1} \Lambda^{4}$ as the topological susceptibility $\kappa_{1} \Lambda^{4} / n_{a}^{2} \sim(0.0756 \mathrm{GeV})^{4}$ [122]. The quality problem can be solved if $\kappa_{2}$ is small enough [12] or induce testable EDM in the proton EDM experiment if $\kappa_{2} \Lambda^{4} \sim(0.3 \mathrm{MeV})^{4}$ [26]. For large enough $\kappa_{3} n^{2}$, we obtain $\epsilon_{a} \sim \mathcal{O}\left(10^{-10}\right) \theta$, which induces the magnetic field of $\mathcal{O}(1)$ aT for $f_{\phi}=10^{8-9} \mathrm{GeV}, \theta=\mathcal{O}(1)$ and $c_{p}=1$. If $a$ and $\phi$ are both non-QCD axions with $\kappa_{1} \sim \kappa_{2} \sim \kappa_{3}, m_{a} \gg$ $m_{\phi}$ can be made by $f_{a} \ll f_{\phi}$. In this case, $\epsilon_{a} \sim \mathcal{O}(\theta)$, which is order 1 for $n_{a}=\mathcal{O}(1)$.

If $\phi$ or $a$ contributes to the density of the Universe (either as DM does or whatever else), as long as it is nonrelativistic, one can approximate

\footnotetext{
${ }^{3}$ This kind of potential is known to lead to a consistent inflation [88,115-121].
}

$$
\{\delta \phi, \delta a\}(t, \vec{x})=\sqrt{\frac{\rho(\vec{x})}{m^{2}}} \cos [m t],
$$

where $\rho(\vec{x})=\left\{\rho_{\phi}(\vec{x}), \rho_{a}(\vec{x})\right\} \quad\left(m=\left\{m_{\phi}, m_{a}\right\}\right)$ represents the density (mass) of $\{\phi, a\}$. We would like to obtain the axion potential from source $J$. Here, we obtain

$$
J=\left\{A_{\phi} \delta \phi^{2}, A_{a} \delta a^{2}\right\}
$$

Then, we can derive the solution to (3) as

$\delta \phi^{\mathrm{ps}}(\vec{x}, t)$

$$
=\left(\frac{1}{4 \pi r} \exp \left(-m_{\phi} r\right)+\frac{\cos \left(r \sqrt{4 m^{2}-m_{\phi}^{2}}\right) \cos (2 m t)}{4 \pi r}\right) .
$$

from a point source of the form

$J^{\mathrm{ps}}(t, \vec{x})=(\cos [m t])^{2} \delta^{3}(\vec{x})=(1+\cos [2 m t]) \delta^{3}(\vec{x})$.

The oscillation term (second term) is not important if we consider $r \gg 1 / m$.

\section{3. $\boldsymbol{C P}$-violating interaction with axion domain wall}

Even if the vacuum is $C P$ conserving, we may still have a topological defect that carries the charge of the long-range force. To see this, let us take $n_{a}=n_{\phi}=1$, in which case the vacuum is $\langle\phi\rangle=\langle a\rangle=0$, i.e., $C P$ conserving. Consider an $a$ domain wall configuration, $a_{\mathrm{DW}}$, which satisfies $a_{\mathrm{DW}}\left[x, y, z \approx z_{\mathrm{DW}}\right] / f_{a} \sim \pi \bmod 2 \pi$ with $z_{\mathrm{DW}}$ being a position for a domain wall stretching in the $x-y$ plane. At other $z$, $a_{\mathrm{DW}} / f_{a}$ takes vacuum value $\langle a\rangle / f_{a} \sim 0 \bmod 2 \pi$.

We can obtain

$$
\frac{\partial V}{\partial \delta \phi} \approx-\kappa_{2} \Lambda^{4} / f_{\phi} \sin \left[a_{\mathrm{DW}} / f_{a}\right]+\mathcal{O}\left(\delta \phi / f_{\phi}\right)
$$

The domain wall width is around $1 / m_{a}$, and thus only within the region $\left|z-z_{\mathrm{DW}}\right| \lesssim 1 / m_{a}$, the rhs $\sim \pm \kappa_{2} \Lambda^{4} / f_{\phi}$, and is otherwise zero. If $1 / m_{\phi} \gtrsim 1 / m_{a}$, we can approximate

$$
\left|\frac{\partial V}{\partial \delta \phi}\right| \sim \delta\left(z-z_{\mathrm{DW}}\right) \frac{\left|\kappa_{2}\right| \Lambda^{4}}{f_{\phi}} \frac{1}{m_{a}} \sim \delta\left(z-z_{\mathrm{DW}}\right) \frac{\sigma_{\mathrm{DW}}}{f_{\phi}}\left|\frac{\kappa_{2}}{\kappa_{1}}\right|
$$

Here, the tension of the domain wall is given by $\sigma_{\mathrm{DW}} \sim f_{a}^{2} m_{a}$. Since $\rho_{\mathrm{DS}}=\sigma_{\mathrm{DW}} \delta\left(z-z_{\mathrm{DW}}\right),|\epsilon| \sim\left|\kappa_{2} / \kappa_{1}\right|$. 


\section{APPENDIX B: INCOHERENT AND COHERENT AVERAGING METHODS}

Using incoherent and coherent averaging, each expected probability distribution for the power/voltage spectrum can be estimated using statistical theory. The incoherent averaging is evaluated as averaging the injected power spectrum. Therefore, it conserves the averaged injected power including noise. On the other hand, coherent averaging is evaluated as averaging each component of the power spectrum and integrating each Fourier component. Therefore, this average method does not preserve injected power but only leaves signals with coherence. In this estimation, the noise spectrum is white. Then, the single time-series noise follows the normal distribution $N\left(0, \sigma^{2}\right)$.

First, the probability distribution of incoherent averaging is estimated as follows. The Fourier transform of $N\left(0, \sigma^{2}\right)$ distribution is separated into the real part and imaginary part. Each component follows the $N\left(0,2 \sigma^{2} / L\right)$, where $L$ is the length of the $x_{i}$. Therefore, the power spectrum of $x_{i}$ will follow the probability distribution $\mathcal{T}_{i} \sim N\left(0,2 \sigma^{2} / L\right)^{2}+$ $N\left(0,2 \sigma^{2} / L\right)^{2}$, which is the scaled chi-square distribution with a degree of freedom of 2 . Then, we average this distribution with repetition number $N$. In symbolic notation,

$$
\begin{aligned}
\mathcal{T} & =\frac{1}{N} \sum_{i}^{N} \mathcal{T}_{i}=\frac{1}{N} \sum_{i=1}^{2 N} N\left(0, \frac{2 \sigma^{2}}{L}\right)^{2} \\
& =\frac{2 \sigma^{2}}{L N} \sum_{i=1}^{2 N} N(0,1)=\frac{2 \sigma^{2}}{L N} \chi_{2 N}^{2} .
\end{aligned}
$$

Therefore, if we normalize the power spectrum to $2 \sigma^{2} /(L N)$, it follows the chi-square distribution with a degree of freedom $2 N$.

The probability distribution for coherent averaging, on the other hand, can be estimated similarly. Coherent averaging conducts the expectation operation to the time series. Therefore, the averaged time series $\langle x\rangle$ follows the distribution $N\left(0, \sigma^{2} / N\right)$. The Fourier transform operation to $\langle x\rangle$ makes power spectrum, and this spectrum will follow the distribution $\mathcal{T}_{c}$ as

$$
\mathcal{T}_{c}=N\left(0, \frac{2 \sigma^{2}}{N L}\right)^{2}=\frac{2 \sigma^{2}}{L N} \chi_{1}^{2}
$$

At the same time, the information about the phase of the signal makes it distinguish the noise element. This implies that a normalized power spectrum of the coherent averaging method follows the chi-square distribution with a degree of freedom 1.

From the derived distribution, the signal to noise ratio can be evaluated explicitly. Assume the injected signal or measured signal forms sinusoidal function with amplitude $\mu$. Then, the distribution of the measured in the presence of white noise follows noncentral chi-square distribution, with noncentrality, $\lambda=\mu^{2} / \alpha$, where $\alpha$ is the normalization constant $\alpha \equiv 2 \sigma^{2} / L N$. The $\chi_{k}^{2}$ distribution has a mean value $k$ and a variance $2 k$. The normalization constant for the power spectrum density is labeled as $\alpha \equiv 2 \sigma^{2} / L N$; then, the SNRs for both averaging methods are calculated as

$$
\begin{aligned}
\mathrm{SNR}_{\mathrm{p}}^{\mathrm{i}} & =\frac{\mu^{2} / \alpha}{\sqrt{4 N}} \\
\mathrm{SNR}_{\mathrm{p}}^{\mathrm{c}} & =\frac{\mu^{2} / \alpha}{\sqrt{2}} .
\end{aligned}
$$

The normalization constant $\alpha \propto 1 / N$; therefore, the SNR has the relationship

$$
\begin{aligned}
& \operatorname{SNR}_{\mathrm{p}}^{\mathrm{i}}=\left(\frac{\lambda}{2}\right) \sqrt{N} \\
& \mathrm{SNR}_{\mathrm{p}}^{\mathrm{c}}=\left(\frac{\lambda}{\sqrt{2}}\right) N,
\end{aligned}
$$

where $\lambda=\mu^{2} L / 2 \sigma^{2}$ is the noncentrality parameter of the noncentral chi-square distribution. Clearly, for $1 \leq N$, the coherent averaging method always has a higher SNR than that of incoherent averaging.

These behaviors can be evaluated numerically. The time-series data $\left(x_{i}\right)$ are composed of sinusoidal signal with a frequency $100 \mathrm{~Hz}$ and amplitude $0.02 \mathrm{~V}_{\mathrm{rms}}$, and $0.2 \mathrm{~V}^{2} / \mathrm{Hz}$ of white noise with a sampling rate $10 \mathrm{kHz}$ is generated. We generated these time-series data $N$ times. Therefore, there are total $N$ sets of the times-series test samples, $X=\left\{x_{1}, x_{2}, \ldots, x_{N}\right\}$. For this time-series dataset $X$, the voltage spectrum is numerically evaluated. Figure 3 shows the two different spectra with $N=10^{4}$. The coherent averaging reduces the noise energy spectrum; therefore, it makes the overall noise floor decrease as expected. On the other hand, the incoherent averaging maintains the noise

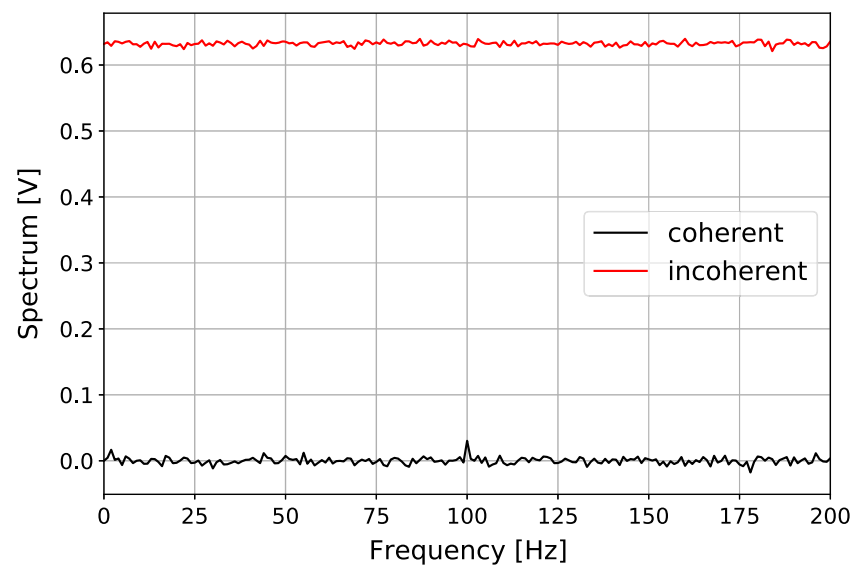

FIG. 3. Simulation of the voltage spectrum depending on the averaging method. 


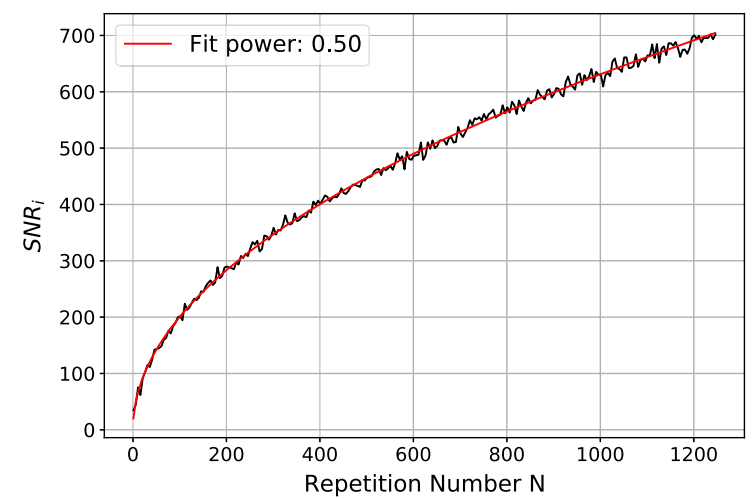

(a)

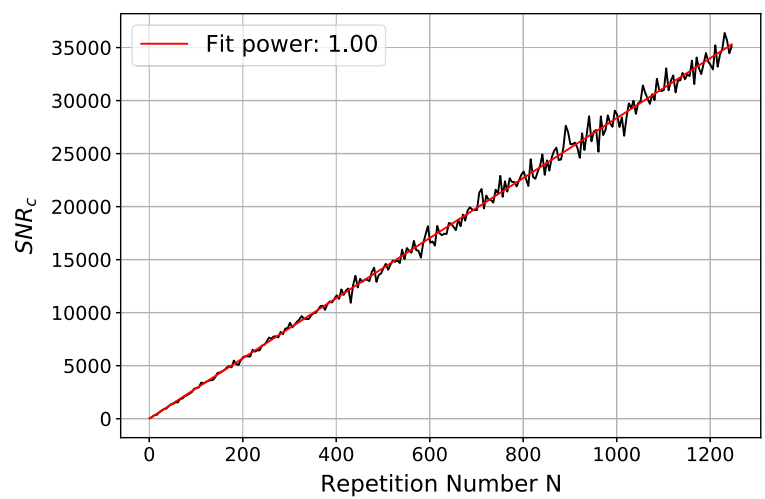

(b)

FIG. 4. (a) SNR dependency to repetition number $N$ for incoherent averaging. (b) SNR dependency to repetition number $N$ for coherent averaging.

energy spectrum, and the averaging process only reduces the fluctuation at the same noise level. Therefore, for the same averaging number, the coherent average has larger SNR than incoherent averaging. The dependency of SNR for repetition number is evaluated with enough high injection signal strength ( $2 \mathrm{~V}_{\text {rms }}$ amplitude sinusoidal wave with $100 \mathrm{~Hz}$ ) to check the relation in Eq. (B4). Figure 4 shows the power SNR dependency to $N$. The calculated SNR (black) is fitted using a model function $f(N)=a N^{b}$, and the fitting parameter $b$ is labeled in Fig. 4. The power SNR of incoherent averaging grows with $\sqrt{N}$, and that of coherent averaging increases with $N$, which showed the expected behavior.

At the same time, the distribution of the field (voltage) can be evaluated for coherent averaging with phase information. Since the axion force injected into the atomic comagnetometer varies with the rotation of the experimental apparatus, the measured signal follows the distribution as $N\left(\mu, 2 \sigma^{2} / L N\right)$. The distribution follows the normal distribution, and the $5 \sigma$ rule is applicable to set the target SNR to reduce the rescanning probability under $0.01 \%$.
[1] R. D. Peccei and H. R. Quinn, $C P$ Conservation in the Presence of Instantons, Phys. Rev. Lett. 38, 1440 (1977).

[2] R. D. Peccei and H. R. Quinn, Constraints imposed by $C P$ conservation in the presence of instantons, Phys. Rev. D 16, 1791 (1977).

[3] S. Weinberg, A New Light Boson?, Phys. Rev. Lett. 40, 223 (1978).

[4] F. Wilczek, Problem of Strong $P$ and $T$ Invariance in the Presence of Instantons, Phys. Rev. Lett. 40, 279 (1978).

[5] E. Witten, Some properties of O(32) superstrings, Phys. Lett. 149B, 351 (1984).

[6] P. Svrcek and E. Witten, Axions in string theory, J. High Energy Phys. 06 (2006) 051.

[7] J.P. Conlon, The QCD axion and moduli stabilisation, J. High Energy Phys. 05 (2006) 078.

[8] A. Arvanitaki, S. Dimopoulos, S. Dubovsky, N. Kaloper, and J. March-Russell, String axiverse, Phys. Rev. D 81, 123530 (2010).

[9] J. Preskill, M. B. Wise, and F. Wilczek, Cosmology of the invisible axion, Phys. Lett. 120B, 127 (1983).

[10] L. F. Abbott and P. Sikivie, A cosmological bound on the invisible axion, Phys. Lett. 120B, 133 (1983).
[11] M. Dine and W. Fischler, The not so harmless axion, Phys. Lett. 120B, 137 (1983).

[12] B. S. Acharya, K. Bobkov, and P. Kumar, An M theory solution to the strong $C P$ problem and constraints on the axiverse, J. High Energy Phys. 11 (2010) 105.

[13] T. Higaki and T. Kobayashi, Note on moduli stabilization, supersymmetry breaking and axiverse, Phys. Rev. D 84, 045021 (2011).

[14] M. Cicoli, M. Goodsell, and A. Ringwald, The type IIB string axiverse and its low-energy phenomenology, J. High Energy Phys. 10 (2012) 146.

[15] R. Daido, T. Kobayashi, and F. Takahashi, Dark matter in axion landscape, Phys. Lett. B 765, 293 (2017).

[16] P. Agrawal and K. Howe, Factoring the strong $C P$ problem, J. High Energy Phys. 12 (2018) 029.

[17] P. W. Graham and A. Scherlis, Stochastic axion scenario, Phys. Rev. D 98, 035017 (2018).

[18] F. Takahashi, W. Yin, and A. H. Guth, QCD axion window and low-scale inflation, Phys. Rev. D 98, 015042 (2018).

[19] M. Demirtas, C. Long, L. McAllister, and M. Stillman, The Kreuzer-Skarke axiverse, J. High Energy Phys. 04 (2020) 138. 
[20] R. T. Co, E. Gonzalez, and K. Harigaya, Axion misalignment driven to the bottom, J. High Energy Phys. 05 (2019) 162.

[21] S.-Y. Ho, F. Takahashi, and W. Yin, Relaxing the cosmological moduli problem by low-scale inflation, J. High Energy Phys. 04 (2019) 149.

[22] H. Matsui, F. Takahashi, and W. Yin, QCD axion window and false vacuum Higgs inflation, J. High Energy Phys. 05 (2020) 154.

[23] T. T. Yanagida, W. Yin, and N. Yokozaki, Bino-wino coannihilation as a prediction in the $E_{7}$ unification of families, J. High Energy Phys. 12 (2019) 169.

[24] F. Takahashi and W. Yin, QCD axion on hilltop by a phase shift of $\pi$, J. High Energy Phys. 10 (2019) 120.

[25] A. Arvanitaki, S. Dimopoulos, M. Galanis, L. Lehner, J. O. Thompson, and K. Van Tilburg, Large-misalignment mechanism for the formation of compact axion structures: Signatures from the QCD axion to fuzzy dark matter, Phys. Rev. D 101, 083014 (2020).

[26] D. J.E. Marsh and W. Yin, Opening the $1 \mathrm{~Hz}$ axion window, J. High Energy Phys. 01 (2021) 169.

[27] S. Nakagawa, F. Takahashi, and W. Yin, Stochastic axion dark matter in axion landscape, J. Cosmol. Astropart. Phys. 05 (2020) 004.

[28] R. Kitano and W. Yin, Strong $C P$ problem and axion dark matter with small instantons, J. High Energy Phys. 07 (2021) 078.

[29] T. W. B. Kibble, Topology of cosmic domains and strings, J. Phys. A 9, 1387 (1976).

[30] T. W. B. Kibble, Some implications of a cosmological phase transition, Phys. Rep. 67, 183 (1980).

[31] F. Takahashi and W. Yin, Kilobyte cosmic birefringence from ALP domain walls, J. Cosmol. Astropart. Phys. 04 (2021) 007.

[32] M. Cicoli, J. P. Conlon, and F. Quevedo, Dark radiation in LARGE volume models, Phys. Rev. D 87, 043520 (2013).

[33] T. Higaki and F. Takahashi, Dark radiation and dark matter in large volume compactifications, J. High Energy Phys. 11 (2012) 125.

[34] J. P. Conlon and M. C. D. Marsh, The cosmophenomenology of axionic dark radiation, J. High Energy Phys. 10 (2013) 214.

[35] A. Hebecker, P. Mangat, F. Rompineve, and L. T. Witkowski, Dark radiation predictions from general large volume scenarios, J. High Energy Phys. 09 (2014) 140.

[36] J. A. Dror, H. Murayama, and N. L. Rodd, The cosmic axion background, Phys. Rev. D 103, 115004 (2021).

[37] J. Jaeckel and W. Yin, The spectrum of dark radiation as a probe of reheating, Phys. Rev. D 103, 115019 (2021).

[38] Y. Minami and E. Komatsu, New Extraction of the Cosmic Birefringence from the Planck 2018 Polarization Data, Phys. Rev. Lett. 125, 221301 (2020).

[39] T. Fujita, K. Murai, H. Nakatsuka, and S. Tsujikawa, Detection of isotropic cosmic birefringence and its implications for axionlike particles including dark energy, Phys. Rev. D 103, 043509 (2021).

[40] V. M. Mehta, M. Demirtas, C. Long, D. J. E. Marsh, L. McAllister, and M. J. Stott, Superradiance in string theory, J. Cosmol. Astropart. Phys. 07 (2021) 033.
[41] S. Nakagawa, F. Takahashi, and M. Yamada, Cosmic birefringence triggered by dark matter domination, arXiv:2103.08153 [Phys. Rev. Lett. (to be published)].

[42] S. M. Carroll, G. B. Field, and R. Jackiw, Limits on a Lorentz and parity violating modification of electrodynamics, Phys. Rev. D 41, 1231 (1990).

[43] S. M. Carroll and G. B. Field, The Einstein equivalence principle and the polarization of radio galaxies, Phys. Rev. D 43, 3789 (1991).

[44] D. Harari and P. Sikivie, Effects of a Nambu-Goldstone boson on the polarization of radio galaxies and the cosmic microwave background, Phys. Lett. B 289, 67 (1992).

[45] S. M. Carroll, Quintessence and the Rest of the World, Phys. Rev. Lett. 81, 3067 (1998).

[46] A. Lue, L.-M. Wang, and M. Kamionkowski, Cosmological Signature of New Parity Violating Interactions, Phys. Rev. Lett. 83, 1506 (1999).

[47] M. Pospelov, A. Ritz, C. Skordis, A. Ritz, and C. Skordis, Pseudoscalar Perturbations and Polarization of the Cosmic Microwave Background, Phys. Rev. Lett. 103, 051302 (2009).

[48] M. A. Fedderke, P. W. Graham, and S. Rajendran, Axion dark matter detection with CMB polarization, Phys. Rev. D 100, 015040 (2019).

[49] P. Agrawal, A. Hook, and J. Huang, A CMB Millikan experiment with cosmic axiverse strings, J. High Energy Phys. 07 (2020) 138.

[50] M. Jain, A. J. Long, and M. A. Amin, CMB birefringence from ultra-light axion string networks, arXiv:2103.10962.

[51] J. Jaeckel and A. Ringwald, The low-energy frontier of particle physics, Annu. Rev. Nucl. Part. Sci. 60, 405 (2010).

[52] A. Ringwald, Exploring the role of axions and other WISPs in the dark Universe, Phys. Dark Universe 1, 116 (2012).

[53] P. Arias, D. Cadamuro, M. Goodsell, J. Jaeckel, J. Redondo, and A. Ringwald, WISPy cold dark matter, J. Cosmol. Astropart. Phys. 06 (2012) 013.

[54] P. W. Graham, I. G. Irastorza, S. K. Lamoreaux, A. Lindner, and K. A. van Bibber, Experimental searches for the axion and axion-like particles, Annu. Rev. Nucl. Part. Sci. 65, 485 (2015).

[55] D. J. E. Marsh, Axion cosmology, Phys. Rep. 643, 1 (2016).

[56] I. G. Irastorza and J. Redondo, New experimental approaches in the search for axion-like particles, Prog. Part. Nucl. Phys. 102, 89 (2018).

[57] L. Di Luzio, M. Giannotti, E. Nardi, and L. Visinelli, The landscape of QCD axion models, Phys. Rep. 870, 1 (2020).

[58] J. E. Moody and F. Wilczek, New macroscopic forces?, Phys. Rev. D 30, 130 (1984).

[59] M. Pospelov, $C P$ odd interaction of axion with matter, Phys. Rev. D 58, 097703 (1998).

[60] E. G. Adelberger, J. H. Gundlach, B. R. Heckel, S. Hoedl, and S. Schlamminger, Torsion balance experiments: A low-energy frontier of particle physics, Prog. Part. Nucl. Phys. 62, 102 (2009).

[61] M. S. Safronova, D. Budker, D. DeMille, D. F. J. Kimball, A. Derevianko, and C. W. Clark, Search for new physics 
with atoms and molecules, Rev. Mod. Phys. 90, 025008 (2018).

[62] G. Raffelt, Limits on a $C P$-violating scalar axion-nucleon interaction, Phys. Rev. D 86, 015001 (2012).

[63] C. A. J. O'Hare and E. Vitagliano, Cornering the axion with $C P$-violating interactions, Phys. Rev. D 102, 115026 (2020).

[64] A. Arvanitaki and A. A. Geraci, Resonantly Detecting Axion-Mediated Forces with Nuclear Magnetic Resonance, Phys. Rev. Lett. 113, 161801 (2014).

[65] A. A. Geraci, G. Rybka, and K. van Bibber (ARIADNE Collaboration), Progress on the ARIADNE axion experiment, Springer Proc. Phys. 211, 151 (2018).

[66] Y. Kahn, B. R. Safdi, and J. Thaler, Broadband and Resonant Approaches to Axion Dark Matter Detection, Phys. Rev. Lett. 117, 141801 (2016).

[67] J. L. Ouellet et al., First Results from ABRACADABRA$10 \mathrm{~cm}$ : A Search for Sub- $\mu \mathrm{eV}$ Axion Dark Matter, Phys. Rev. Lett. 122, 121802 (2019).

[68] P. W. Graham and S. Rajendran, New observables for direct detection of axion dark matter, Phys. Rev. D 88, 035023 (2013).

[69] D. Budker, P. W. Graham, M. Ledbetter, S. Rajendran, and A. Sushkov, Proposal for a Cosmic Axion Spin Precession Experiment (CASPEr), Phys. Rev. X 4, 021030 (2014).

[70] G. Carosi and G. Rybka, Overview of the cosmic axion spin precession experiment (CASPEr), Springer Proc. Phys. 245, 105 (2020).

[71] A. Garcon et al., The cosmic axion spin precession experiment (CASPEr): A dark-matter search with nuclear magnetic resonance, arXiv:1707.05312.

[72] T. Wu et al., Search for Axionlike Dark Matter with a Liquid-State Nuclear Spin Comagnetometer, Phys. Rev. Lett. 122, 191302 (2019).

[73] Y. V. Stadnik and V. V. Flambaum, Axion-induced effects in atoms, molecules, and nuclei: Parity nonconservation, anapole moments, electric dipole moments, and spingravity and spin-axion momentum couplings, Phys. Rev. D 89, 043522 (2014).

[74] C. Abel et al., Search for Axionlike Dark Matter through Nuclear Spin Precession in Electric and Magnetic Fields, Phys. Rev. X 7, 041034 (2017).

[75] S. Pustelny et al., The global network of optical magnetometers for exotic physics (GNOME): A novel scheme to search for physics beyond the standard model, Ann. Phys. (Amsterdam) 525, 659 (2013).

[76] S. Afach et al., Characterization of the global network of optical magnetometers to search for exotic physics (GNOME), Phys. Dark Universe 22, 162 (2018).

[77] S. Afach et al., Search for topological defect dark matter using the global network of optical magnetometers for exotic physics searches (GNOME), arXiv:2102.13379.

[78] S. Schlamminger, K. Y. Choi, T. A. Wagner, J. H. Gundlach, and E. G. Adelberger, Test of the Equivalence Principle Using a Rotating Torsion Balance, Phys. Rev. Lett. 100, 041101 (2008).

[79] Y. V. Stadnik and V. V. Flambaum, Improved limits on interactions of low-mass spin-0 dark matter from atomic clock spectroscopy, Phys. Rev. A 94, 022111 (2016).
[80] P. Touboul et al., MICROSCOPE Mission: First Results of a Space Test of the Equivalence Principle, Phys. Rev. Lett. 119, 231101 (2017).

[81] M. Pospelov, A. Ritz, and M. B. Voloshin, Bosonic superWIMPs as keV-scale dark matter, Phys. Rev. D 78, 115012 (2008).

[82] K. Nakayama, F. Takahashi, and T. T. Yanagida, Anomalyfree flavor models for Nambu-Goldstone bosons and the $3.5 \mathrm{keV}$ X-ray line signal, Phys. Lett. B 734, 178 (2014).

[83] F. Takahashi, M. Yamada, and W. Yin, XENON1T Excess from Anomaly-Free Axionlike Dark Matter and Its Implications for Stellar Cooling Anomaly, Phys. Rev. Lett. 125, 161801 (2020).

[84] I. M. Bloch, A. Caputo, R. Essig, D. Redigolo, M. Sholapurkar, and T. Volansky, Exploring new physics with $\mathrm{O}(\mathrm{keV})$ electron recoils in direct detection experiments, J. High Energy Phys. 01 (2021) 178.

[85] T. Li, The KSVZ axion and Pseudo-Nambu-Goldstone boson models for the XENON1T excess, arXiv:2007 .00874 .

[86] P. Athron et al., Global fits of axion-like particles to XENON1T and astrophysical data, J. High Energy Phys. 05 (2021) 159.

[87] C. Han, M. L. López-Ibáñez, A. Melis, O. Vives, and J. M. Yang, Anomaly-free leptophilic axionlike particle and its flavor violating tests, Phys. Rev. D 103, 035028 (2021).

[88] F. Takahashi, M. Yamada, and W. Yin, What if ALP dark matter for the XENON1T excess is the inflaton, J. High Energy Phys. 01 (2021) 152.

[89] P. W. Graham, S. Hacomeroglu, D. E. Kaplan, Z. Omarov, S. Rajendran, and Y. K. Semertzidis, Storage ring probes of dark matter and dark energy, Phys. Rev. D 103, 055010 (2021).

[90] L. Visinelli, S. Baum, J. Redondo, K. Freese, and F. Wilczek, Dilute and dense axion stars, Phys. Lett. B 777, 64 (2018).

[91] J. F. Navarro, C. S. Frenk, and S. D. M. White, The structure of cold dark matter halos, Astrophys. J. 462, 563 (1996).

[92] M. Cirelli, G. Corcella, A. Hektor, G. Hutsi, M. Kadastik, P. Panci, M. Raidal, F. Sala, and A. Strumia, PPPC 4 DM ID: A poor particle physicist cookbook for dark matter indirect detection, J. Cosmol. Astropart. Phys. 03 (2011) 051.

[93] A. Burkert, The structure of dark matter halos in dwarf galaxies, Astrophys. J. Lett. 447, L25 (1995).

[94] P. Salucci and A. Burkert, Dark matter scaling relations, Astrophys. J. Lett. 537, L9 (2000).

[95] P. Salucci, The distribution of dark matter in galaxies, Astron. Astrophys. Rev. 27, 2 (2019).

[96] N. Aghanim et al. (Planck Collaboration), Planck 2018 results. VI. Cosmological parameters, Astron. Astrophys. 641, A6 (2020).

[97] R. Hlozek, D. Grin, D. J. E. Marsh, and P. G. Ferreira, A search for ultralight axions using precision cosmological data, Phys. Rev. D 91, 103512 (2015). 
[98] R. Mayle, J. R. Wilson, J. R. Ellis, K. A. Olive, D. N. Schramm, and G. Steigman, Constraints on axions from SN 1987a, Phys. Lett. B 203, 188 (1988).

[99] G. Raffelt and D. Seckel, Bounds on Exotic Particle Interactions from SN 1987a, Phys. Rev. Lett. 60, 1793 (1988).

[100] M. S. Turner, Axions from SN 1987a, Phys. Rev. Lett. 60, 1797 (1988).

[101] J. H. Chang, R. Essig, and S. D. McDermott, Supernova 1987A constraints on Sub-GeV dark sectors, millicharged particles, the QCD Axion, and an axion-like particle, J. High Energy Phys. 09 (2018) 051.

[102] N. Viaux, M. Catelan, P. B. Stetson, G. Raffelt, J. Redondo, A. A. R. Valcarce, and A. Weiss, Neutrino and Axion Bounds from the Globular Cluster M5 (NGC 5904), Phys. Rev. Lett. 111, 231301 (2013).

[103] F. Capozzi and G. Raffelt, Axion and neutrino bounds improved with new calibrations of the tip of the red-giant branch using geometric distance determinations, Phys. Rev. D 102, 083007 (2020).

[104] M. Pospelov and A. Ritz, Electric dipole moments as probes of new physics, Ann. Phys. (Amsterdam) 318, 119 (2005).

[105] B. J. Venema, P. K. Majumder, S. K. Lamoreaux, B. R. Heckel, and E. N. Fortson, Search for a Coupling of the Earth's Gravitational Field to Nuclear Spins in Atomic Mercury, Phys. Rev. Lett. 68, 135 (1992).

[106] L. Ackerman, M. R. Buckley, S. M. Carroll, and M. Kamionkowski, Dark matter and dark radiation, Phys. Rev. D 79, 023519 (2009).

[107] Y. B. Zeldovich, I. Y. Kobzarev, and L. B. Okun, Cosmological consequences of the spontaneous breakdown of discrete symmetry, Zh. Eksp. Teor. Fiz. 67, 3 (1974), http:// jetp.ras.ru/cgi-bin/e/index/r/67/1/p3?a=list.

[108] A. Vilenkin, Cosmic strings and domain walls, Phys. Rep. 121, 263 (1985).

[109] T. W. Kornack, R. K. Ghosh, and M. V. Romalis, Nuclear Spin Gyroscope Based on an Atomic Comagnetometer, Phys. Rev. Lett. 95, 230801 (2005).
[110] J. M. Brown, S. J. Smullin, T. W. Kornack, and M. V. Romalis, New Limit on Lorentz- and cpt-Violating Neutron Spin Interactions, Phys. Rev. Lett. 105, 151604 (2010).

[111] T. W. Kornack and M. V. Romalis, Dynamics of Two Overlapping Spin Ensembles Interacting by Spin Exchange, Phys. Rev. Lett. 89, 253002 (2002).

[112] D. F. Jackson Kimball, J. Dudley, Y. Li, S. Thulasi, S. Pustelny, D. Budker, and M. Zolotorev, Magnetic shielding and exotic spin-dependent interactions, Phys. Rev. D 94, 082005 (2016).

[113] A. Hook and J. Huang, Probing axions with neutron star inspirals and other stellar processes, J. High Energy Phys. 06 (2018) 036.

[114] T. Kumar Poddar, S. Mohanty, and S. Jana, Constraints on ultralight axions from compact binary systems, Phys. Rev. D 101, 083007 (2020).

[115] M. Czerny and F. Takahashi, Multi-natural inflation, Phys. Lett. B 733, 241 (2014).

[116] M. Czerny, T. Higaki, and F. Takahashi, Multi-natural inflation in supergravity and BICEP2, Phys. Lett. B 734, 167 (2014).

[117] D. Croon and V. Sanz, Saving natural inflation, J. Cosmol. Astropart. Phys. 02 (2015) 008.

[118] T. Higaki and F. Takahashi, Elliptic inflation: Interpolating from natural inflation to $\mathrm{R}^{2}$-inflation, J. High Energy Phys. 03 (2015) 129.

[119] R. Daido, F. Takahashi, and W. Yin, The ALP miracle: Unified inflaton and dark matter, J. Cosmol. Astropart. Phys. 05 (2017) 044.

[120] R. Daido, F. Takahashi, and W. Yin, The ALP miracle revisited, J. High Energy Phys. 02 (2018) 104.

[121] F. Takahashi and W. Yin, ALP inflation and Big Bang on Earth, J. High Energy Phys. 07 (2019) 095.

[122] S. Borsanyi et al., Calculation of the axion mass based on high-temperature lattice quantum chromodynamics, Nature (London) 539, 69 (2016). 